FORMATION Formation emploi

Revue française de sciences sociales

118 | avril-juin 2012

10 ans de parcours professionnels des jeunes :

l'intérêt des études longitudinales

\title{
Peut-on en finir avec le déclassement ? : Évolution du déclassement dans la fonction publique en début de carrière
}

Can we put an end to overducation?: The overeducation dynamics during the early careers in public sector

Ist eine niedrigere Einstufung noch angebracht?: Entwicklung der niedrigeren beruflichen Einstufung zu Beginn der Berufslaufbahn im öffentlichen Dienst ¿Se puede terminar con el desclasamiento ? Evolución del desclasamiento en la función pública al inicio de carrera

Vanessa di Paola et Stéphanie Moullet

\section{OpenEdition Journals}

Édition électronique

URL : http://journals.openedition.org/formationemploi/3610

DOI : 10.4000/formationemploi.3610

ISSN : 2107-0946

Éditeur

La Documentation française

Édition imprimée

Date de publication : 30 juin 2012

Pagination : 83-101

ISSN : 0759-6340

Référence électronique

Vanessa di Paola et Stéphanie Moullet, « Peut-on en finir avec le déclassement ? : Évolution du déclassement dans la fonction publique en début de carrière ", Formation emploi [En ligne], 118 | avriljuin 2012, mis en ligne le 19 juillet 2012, consulté le 30 octobre 2020. URL : http://

journals.openedition.org/formationemploi/3610; DOI : https://doi.org/10.4000/formationemploi.3610 


\title{
Peut-on en finir avec le déclassement ?
}

\section{Évolution du déclassement dans la fonction publique en début de carrière}

\author{
Vanessa dI PAOLA \\ Maître de conférence en économie, Aix-Marseille université, \\ CNRS - Laboratoire d'économie et de sociologie du travail (LEST UMR 6123)
}

Stéphanie Moullet

Maître de conférence en économie, Aix-Marseille université, CNRS - Laboratoire d'économie et de sociologie du travail (LEST UMR 6123) et Institut Régional du Travail (IRT)

Résumé

Peut-on en finir avec le déclassement ? Évolution du déclassement dans la fonction publique en début de carrière

Notre travail porte sur la dynamique du déclassement au cours des débuts de carrière dans la fonction publique. Parce qu'elle permet de couvrir les dix premières années de vie active, la quatrième interrogation de l'enquête du Céreq, Génération 98, en 2008, permet d'observer le caractère transitoire ou durable du déclassement selon le statut d'entrée dans l'emploi - fonctionnaire ou non - et le sexe. On constate une inertie des situations de déclassement statistique, et ce plus particulièrement pour les femmes, ainsi qu'un double mouvement de reclassement et acquisition du statut de fonctionnaire. La confrontation des indicateurs statistique et subjectif de déclassement révèle la non-correspondance des deux dimensions.

Mots clés : Déclassement professionnel $\bullet$ cheminement professionnel $\bullet$ insertion professionnelle $\bullet$ fonction publique $\bullet$ femme $\bullet$ enquête génération 1998

Abstract

Can we put an end to overducation? The overeducation dynamics during the early careers in public sector

Our work focuses on the overeducation dynamics during the early careers in public sector. We use the fourth wave of Generation 98 survey, which covers the ten first years of the working life of 1998 school leavers. We examine the overeducation transition depending on the labor market entrance status - civil servant or not - and the gender. We find that overeducation is not a temporary phenomenon; especially for women and that 
the exit from overeducation state is easier while becoming civil servant. A comparison between statistical measurement and subjective measurement of overeducation reveals the mismatch of the two dimensions of this phenomenon.

Key words: Demotion $\bullet$ occupational paths $\bullet$ transition from school to work $\bullet$ civil service - woman $\bullet$ generation 98 survey

Journal of Economic Literature: J 24, J 45

Traduction : Auteurs

Au cours des dernières décennies, le décalage entre le niveau de diplôme requis pour exercer un emploi et celui détenu par les jeunes débutants est apparu comme un phénomène majeur. Dés lors, ce relâchement du lien diplôme - qualification de l'emploi (Baudelot et Glaude, 1989 ; Vinokur, 1995), et sa conséquence, le déclassement, ont donné lieu à de nombreux travaux, en particulier en France (Forgot Gautié, 1997 ; Battu et al., 2000 ; Giret et Lemistre, 2004).

D’un point de vue méthodologique, les travaux récents relatifs au déclassement font référence à deux mesures : l'approche objective ou statistique, qui confronte la nomenclature de PCS (professions et catégories sociales) des emplois occupés à celle des diplômes, et l'approche subjective, basée, pour sa part, sur le ressenti des individus ${ }^{1}$. Après trois ans de vie active, près d'un jeune actif occupé sur cinq est déclassé (Giret et al., 2006). Cette proportion est d'autant plus marquée que l'emploi se situe dans le secteur privé plutôt que dans la fonction publique (di Paola et Moullet, 2009).

Pour autant, la fonction publique présente un ensemble de caractéristiques qui fait d'elle un champ d'analyse du déclassement particulièrement pertinent. En effet, elle concentre une population salariée plus diplômée que dans le secteur privé : parmi les jeunes sortis de formation initiale en 2001-2002, près de $38 \%$ des titulaires d'une licence ou plus y sont entrés contre $3 \%$ des non-diplômés (Audier et alii, 2004). De plus, la fonction publique est d'autant plus attractive que la conjoncture économique est dégradée : la recherche de sécurité de l'emploi se lit au travers de l'influence positive et significative du taux de chômage des jeunes sur le nombre de candidats aux concours (Fougère et Pouget, 2004). Ainsi, l'attractivité manifeste de la fonction publique et la proportion plus élevée de diplômés que dans le secteur privé font que les lauréats aux concours sont largement surqualifiés : pour les concours de catégories $\mathrm{B}$ et $\mathrm{C}$ (respectivement niveaux IV et $\mathrm{V}$ de formation - Baccalauréat et CAP-BEP) par exemple, trois lauréats sur quatre ont un diplôme supérieur à celui exigé (Rapport annuel de l'Observatoire de l'emploi public,

1. Il existe une troisième approche, normative, supposant une relation formation-emploi définie a priori à travers une table de correspondance diplôme-PCS. Notamment parce que la seule table disponible en France est trop ancienne (Affichard, 1981), cette mesure n'est plus retenue ( $c f$. partie 2.2). 
2004-2005). Les analyses conduites à ce sujet, sur les débuts de vie active, ont largement mis en évidence l'ampleur du phénomène de déclassement au sein de la fonction publique (di Paola et alii, 2005).

Les différents travaux sur l'insertion professionnelle étudient certes la question du déclassement à un moment du temps ; toutefois, en France, à notre connaissance, aucun n'examine la dynamique du phénomène ${ }^{2}$. Pourtant, la dimension transitoire ou non du déclassement est au centre des travaux théoriques. L’enquête 2008 du Céreq interroge, pour la quatrième fois, les sortants de la génération 1998. Elle couvre les dix premières années de vie active et rend désormais possible l'observation des reclassements éventuels.

Pour éclairer l'analyse, on ne peut ignorer à la fois la féminisation de la fonction publique et la coexistence du statut de fonctionnaire et de non-titulaire ${ }^{3}$. En effet, de manière générale, les jeunes femmes sont, sur le marché du travail, plus exposées au risque de déclassement à l'embauche. Or, au sein de la fonction publique, nos précédents travaux (di Paola et Moullet, 2009) ont montré qu'il n'existe pas d'écarts significatifs du déclassement à l'embauche entre jeunes hommes et jeunes femmes. Pour autant, existe-t-il, dans ce secteur d'emploi présentant pour elles une assurance contre les aléas du marché privé, (conditions de travail meilleures, moindre discrimination à l'embauche, discrimination salariale limitée, choix de métiers,...) un frein plus grand à la sortie de la situation de déclassement initial ? Autrement dit, les hommes rattrapent-ils plus vite une situation de déclassement initiale, profitant d'une progression de carrière plus favorable que celle des femmes $?^{4}$ Notre analyse s'appuiera essentiellement sur de la statique comparative et non pas sur les transitions individuelles, en mettant en regard les situations détenues par les jeunes en 2001 avec celles détenues 2008 .

Par ailleurs, on a également montré que si les femmes ne sont pas significativement plus déclassées que les hommes, elles ont moins fréquemment le sentiment de l'être. Autrement dit, des situations identiques de déclassement ne donnent pas lieu au même ressenti pour les populations des deux sexes 5 . Dans la mesure où l'on traite de la dimension sexuée pour

2. Soulignons cependant l'article de Nauze-Fichet et Tomasini (2005) qui traite des trajectoires de reclassement salarial sur les trois premières années de vie active, et le travail de Rubb (2003) sur données américaines.

3. Rappelons que $15 \%$ des emplois occupés dans la fonction publique le sont par des non-titulaires (Rapport annuel de l'Observatoire de l'emploi public, 2004-2005).

4. On connaît le phénomène du plafond de verre (McDowell et alii, 1999), empêchant ou limitant l'accès des femmes aux postes à responsabilité, sous forme de barrière invisible; ce phénomène, rebaptisé « ciel de plomb» par Catherine Mary dans une étude portant sur les évolutions de carrières des hommes et des femmes au CNRS (mission pour la place des femmes au CNRS, 2001-2004), existe dans la fonction publique comme dans le secteur privé.

5. Ce qui a été traduit dans un précédent article (di Paola, Moullet, 2009) par l'existence d'un plus grand " consentement à payer " pour les femmes. 
décrire la dynamique du déclassement, on enrichira l'analyse de la confrontation des indicateurs statistiques et subjectifs du déclassement.

Enfin, les jeunes en emploi dans la fonction publique n'accèdent pas tous à l'emploi à vie dès leur embauche. Il s’agit donc de savoir comment le déclassement des fonctionnaires, par le biais des concours internes, évolue, et de comparer cette progression avec les trajectoires de reclassement des non-titulaires.

La première partie aborde donc les arguments théoriques du caractère transitoire ou non du déclassement. La partie suivante décrit les données utilisées et les indicateurs retenus. Les évolutions des situations de déclassement dans la fonction publique en début de vie active, selon le statut et le sexe, sont mesurées dans la partie qui suit. Enfin, dans une dernière partie, nous étudions la correspondance entre les mesures objectives et statistiques du phénomène, ainsi que son évolution, et ce au regard du statut d'emploi et du sexe.

\section{Appréhender le déclassement}

Le déclassement fait l'objet de débat dans la littérature et, notamment, son caractère transitoire ou durable n'est pas tranché dans les explications théoriques. Nous ferons état ici de différentes approches. Par ailleurs, la notion même de déclassement peut renvoyer à plusieurs dimensions : normative, statistique ou subjective, autant de façons de mesurer le déclassement que nous aborderons ensuite.

\subsection{Les débats théoriques : une situation transitoire ou durable ?}

D'un point de vue théorique, le déclassement est envisagé comme transitoire pour certains auteurs, durable pour d'autres. En effet, dans le cadre de la théorie du capital humain, le déclassement correspond à une situation temporaire où les entreprises n'utilisent pas pleinement les qualifications des salariés, ni ne les rémunèrent à leur productivité marginale potentielle. Cette théorie peut également expliquer le déclassement comme le résultat d'un choix délibéré : l'emploi de bas niveau constitue un moyen d'investir en capital humain spécifique ${ }^{6}$ (Hartog, 1999). En accord avec cette dernière interprétation, Sicherman (1991) met en évidence une probabilité de mobilité professionnelle ascendante accrue, par promotion interne ou changement d'entreprise, pour les individus déclassés. Dans cette configuration, l'hypothèse qui prévaut est celle d'un rattrapage d'un déclassement initial.

6. Le capital humain général est non spécialisé et transférable d'une entreprise à l'autre, le capital humain spécifique est lié à des compétences propres à une entreprise. Si le jeune est doté en capital humain général de manière " excédentaire " par rapport aux exigences de l'emploi, en occupant un emploi déclassé, le jeune peut acquérir du capital humain spécifique et par là, espérer se reclasser au sein de l'entreprise qui l'emploie. 
De manière alternative, le déclassement peut devenir une situation durable relevant d'un phénomène de dépendance d'état. Selon Mc Cormick (1990), en situation d'information imparfaite, l'employeur considère l'information relative au dernier emploi occupé par l'individu comme signal de sa productivité. Une situation d'emploi déclassé est identifiée comme signal d'une productivité moindre. Ainsi, l'état dans lequel se trouve l'individu prime sur son diplôme ; en conséquence, si l'individu ne sort pas rapidement de la situation de déclassement initial, il pourrait s'y trouver enfermé car plus le temps passe, moins le niveau d'études compte. Enfin, le déclassement peut également devenir persistant, si la structure de qualification des emplois n'évolue pas aussi vite que l'offre de diplômés (Tsang et Levin, 1985). Par son mode de gestion de la main-d'œuvre où la progression

\section{Encadré 1 \\ La Génération 98 et ses ré-interrogations}

L'analyse des évolutions des situations de déclassement est conduite à partir des enquêtes Génération 98 produites par le Céreq. Elle comporte quatre interrogations successives, en 2001, 2003, 2005 et 2008. Elle interroge, à chacune de ces dates, une population de jeunes sortis du système éducatif en 1998, représentative de l'ensemble des sortants de tous niveaux et spécialités de formation initiale en France métropolitaine. II s'agit d'une enquête rétrospective, les individus interrogés décrivant mois par mois la situation dans laquelle ils se trouvent (emploi, chômage, inactivité...) depuis leur sortie de formation initiale jusqu'à la date d'interrogation.

La quatrième interrogation, au printemps 2008, permet quant à elle d'élargir la fenêtre d'observation au-delà de l'entrée dans la vie active ; de même, elle éclaire les débuts de carrière en essayant de saisir certains déterminants qui président à leur différenciation. À cette étape, la population interrogée pour la quatrième fois est restreinte à 11000 individus, contre près de 55000 jeunes enquêtés en 2001. Couvrant les dix premières années de vie active, l'enquête permet de dépasser la phase d'insertion; elle permet ainsi d'observer les éventuels rattrapages du déclassement initial.

Afin d'obtenir des temporalités comparables pour tous les individus, nous considérons les situations d'emploi à la première et à la dernière date d'enquête. La population étudiée est restreinte aux jeunes en emploi dans la fonction publique, en 2001, et en emploi, quel que soit le secteur, en 2008 , soit $18,5 \%$ de la génération des jeunes sortis du système éducatif en 1998 (soit 14,3\% des hommes de la génération et $23 \%$ des femmes).

Près de deux jeunes sur dix en emploi dans la fonction publique en 2001 sont passés dans le secteur privé en 2008. II s'agit davantage de femmes que d'hommes (57\%). Lorsqu'ils sont fonctionnaires en 2001 , le taux de départ de la fonction publique est plus faible pour les femmes: $7 \%$ contre $12 \%$ pour leurs homologues masculins.

Concernant la population étudiée toujours - les jeunes travaillant dans la fonction publique en 2001 et actifs occupés en 2008 - on compte $63 \%$ de jeunes femmes. À l'embauche de l'emploi occupé en $2001,37 \%$ sont fonctionnaires ( $43 \%$ pour les jeunes hommes et $34 \%$ pour leurs consœurs). À la date d'interrogation, en 2001 , le nombre de fonctionnaires a largement augmenté pour atteindre respectivement $60 \%$ pour les hommes et $56 \%$ pour les femmes (cf. tableau 1).

Au final, en 2008 , on compte près de $78 \%$ de fonctionnaires ( $80 \%$ de la population féminine, soit un taux supérieur de 4 points à celui des hommes). 
professionnelle est soumise à l'ancienneté ou à la réussite aux concours internes, la fonction publique apparaît comme le secteur d'emploi le plus sujet à l'inertie de sa structure d'emploi. Elle est aussi le lieu d'emploi d'une main-d'œuvre particulièrement diplômée.

Au-delà des situations statistiques de déclassement, comment les ressentis évoluent-ils pour les deux sexes? On sait que la satisfaction que les hommes et les femmes tirent de leur activité professionnelle ne repose pas sur les mêmes déterminants ; ils n’attachent notamment pas la même importance à leur rémunération (Baudelot et alii, 2003). En conséquence, à situation statistique donnée, les femmes s'estiment moins déclassées que les hommes dans la même configuration; un résultat qui va dans le sens d'une plus grande satisfaction des femmes dans l'emploi, à situation identique (di Paola et Moullet, 2009).

De plus, le sentiment de déclassement peut également évoluer sans que son niveau statistique ou même que le statut d'emploi ne soient modifiés, du fait du phénomène des préférences adaptatives (Sen 1992, Elster, 1983). Il s'agit d'une prédisposition selon laquelle les individus s'adaptent à leur situation et comparent leur état à un niveau d'aspiration. $\mathrm{Si}$ ce dernier niveau se modifie dans le temps, le ressenti du déclassement s'en trouvera modifié en conséquence : dès lors, l'acceptation du déclassement avec le temps (et donc la disparition de tout ressenti à ce sujet) est-elle plus fréquente dans la fonction publique pour les femmes que pour les hommes?

Par ailleurs, des travaux sur l'articulation entre déclassement statistique et satisfaction professionnelle corroborent le caractère non transitoire du déclassement. En effet, Tansg et al. (1991) mettent en évidence un effet négatif du déclassement sur la satisfaction au travail et par là, de façon indirecte, sur la productivité ; ils ne déterminent toutefois pas si l'effet du surplus éducatif sur la satisfaction relève d'attentes non satisfaites ou des compétences sous-utilisées. Etant moins productifs, les salariés déclassés ont des chances moindres d'accéder à des emplois correspondant à leur niveau de qualification.

\subsection{Mesurer le déclassement : approches statistique ou subjective}

Mesurer le déclassement suppose de définir la population des déclassés. Est considérée comme déclassée toute personne dont le niveau de formation ne correspond pas au niveau " normalement » requis pour l'emploi qu'elle occupe. Usuellement, trois définitions sont retenues : l'approche normative, l'approche statistique et l'approche subjective (Giret 2005 ; di Paola et alii, 2005).

L'approche normative, trop datée, n'est pas retenue ici dans la mesure où elle repose sur des analyses détaillées des contenus d'emploi et de formation qui n’ont plus été reproduites en France depuis le travail d'Affichard en 1981'. Concernant cette approche normative, dans une étude antérieure (di Paola et alii, 2005) portant exclusivement sur les jeunes en

7. Soulignons que, de surcroît, ce travail ne porte que sur le secteur privé. 
emploi dans la fonction publique d'État et territoriale, nous avons retenu la définition statutaire du diplôme minimal requis pour l'accès aux emplois de différentes catégories. Nous avons mis en évidence des taux de déclassement très élevés (de l'ordre de $89 \%$ pour les fonctionnaires de la fonction publique territoriale et plus de $60 \%$ pour ceux de la fonction publique d'État), attribuant un caractère absorbant à une telle mesure ${ }^{8}$. Cette approche simple du déclassement normatif n'est pas satisfaisante. Elle met notamment en évidence des requalifications statistiques des contenus de travail non prises en compte dans la correspondance entre diplômes exigés au niveau des concours et emplois. Ces informations existent inévitablement ; pour autant, leur recensement pour tous les métiers de la fonction publique, afin de mettre à jour les correspondances diplômes-contenu des emplois, reste une entreprise considérable, en particulier dans une démarche quantitative.

Le déclassement qualifié de statistique renvoie à une norme statistique. En effet, le niveau de formation "normalement " requis pour occuper un poste est défini par rapport au niveau de diplôme du plus grand nombre de personnes occupant ce type d'emploi. Cette mesure s'appuie sur les tableaux de contingence croisant diplômes et catégories socioprofessionnelles. Elle repose sur une analyse des écarts à l'indépendance (Forgeot et Gautié 1997 ; Nauze-Fichet et Tomasini, 2001). L'indicateur est sensible au découpage retenu de ces deux variables. Les niveaux de diplôme ici retenus sont : non qualifiés, CAP ou BEP (respectivement certificat d'aptitude professionnelle et brevet d'études professionnelles), $\mathrm{Bac}, \mathrm{Bac}+2,2^{\text {ème }}$ cycle et $3^{\text {ème }}$ cycle de l'enseignement supérieur ; les catégories socioprofessionnelles correspondent à : ouvriers non qualifiés, employés non qualifiés, ouvriers qualifiés, employés qualifiés, techniciens, professions intermédiaires et cadres.

La dimension salariale du déclassement peut également être mesurée, et ce avec les mêmes distinctions, normatives, statistiques et subjectives. Cet aspect du déclassement nécessite une attention particulière et n'a pas été retenu ici (voir sur ce point di Paola, Moullet, 2009).

La valeur de l'indicateur statistique est totalement contingente à la population sur laquelle il est construit. Ici, la population servant à identifier les situations de correspondances " normales " entre formation et emploi est celle des jeunes en emploi dans la fonction publique en 2001, et quel que soit leur secteur d'emploi en 2008. Lorsque la population de référence est celle en emploi indépendamment du secteur, les taux de déclassement statistique qui en découlent ne sont pas les mêmes. De plus, puisque l'on cherche à appréhender des évolutions du déclassement, à quelle correspondance formation-emploi doit-on se référer : celle de 2001 ou celle de 2008 ? Nous avons choisi de retenir une norme correspondant à l'année d'observation : le déclassement est identifié relativement aux situations d'emploi en 2001 et en 2008. Ce choix permet d'internaliser les mobilités professionnelles au sein de l'indicateur ; ainsi, des situations de déclassement en 2001 peuvent être qualifiées de «normales » en 2008 et inversement.

8. Tous les individus déclassés statistiquement le sont systématiquement d'un point de vue normatif. 
Le déclassement subjectif, quant à lui, renvoie à une norme personnelle, à un ressenti. On se réfère à l'auto appréciation des jeunes vis-à-vis de leur situation, à travers la réponse à la question: "A propos de cet emploi, diriez-vous que vous étiez utilisé (a) à votre niveau de compétences, (b) en dessous de votre niveau de compétences, (c) au-dessus de votre niveau de compétences?» Avoir le sentiment d'être employé en dessous de son niveau de compétences correspond alors à un déclassement subjectif.

Les individus, lorsqu'ils s'expriment sur l'usage de leurs compétences, ne se centrent probablement pas sur la correspondance entre formation et emploi. En effet, les compétences renvoient de manière seulement indirecte aux deux dimensions mobilisées pour définir le déclassement statistique ou normatif, à savoir la correspondance entre le niveau de qualification à l'issue de la formation initiale et le niveau de qualification de l'emploi occupé, lu à travers les PCS. En outre, pour l'individu interrogé, d'autres dimensions relatives à la satisfaction professionnelle peuvent contribuer à alimenter ce sentiment de déclassement. Toutefois, "l'approche subjective présente l'intérêt de mieux prendre en compte l'environnement du salarié et de s'affranchir des problèmes de nomenclature d'emploi " (Giret, 2005, p. 280).

En définitive, les approches statistiques et subjectives retenues, l'une comme l'autre, tiennent compte de la situation de chaque individu relativement aux situations des autres. En effet, être statistiquement déclassé signifie ne pas être dans la situation que connaît la majorité de l'échantillon de jeunes considérés (de même niveau de formation initiale). De manière semblable, le sentiment de déclassement de chacun des jeunes est construit en référence à son entourage, par rapport à un niveau jugé moyen dans l'entreprise dans laquelle il est employé, ou bien par rapport à un niveau moyen dans un sous-groupe de référence (groupe d'amis, famille, etc.). Ce qui fonde le sentiment de déclassement évolue dans le temps et ce qui fait référence en tout début de vie active est certainement moins opératoire après plusieurs années d'expérience professionnelle.

La partie suivante dresse un état des lieux des situations de déclassement et de leurs évolutions.

\section{2) Le déclassement: une situation qui dure}

En 2001, trois ans après la fin de leur formation initiale, 15,9 \% des jeunes en emploi dans la fonction publique sont statistiquement déclassés ; sept ans plus tard, ils sont encore $14,3 \%$ à être dans cette situation, faisant du déclassement un état durable au-delà de la phase d'insertion. Au cours de cette période, la conjoncture économique s'est largement dégradée, pour les jeunes en particulier : le taux de chômage des moins de 25 ans passe en effet de $16,1 \%$ en 2001 à 20,7 \% en 2008. La hausse du taux de chômage est un des facteurs importants expliquant le développement du déclassement, notamment à travers le phénomène de file d'attente; ce phénomène consiste en un report des diplômés au 
Tableau 1. Taux de déclassement statistique (\%)

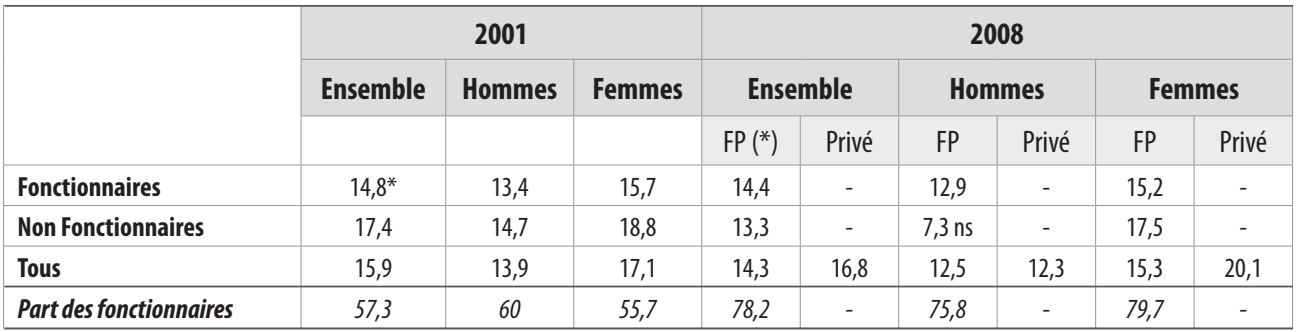

Source : Enquête Céreq 2008 auprès de la génération $98,4^{\text {ème }}$ interrogation.

Champ : jeunes en emploi dans la fonction publique en 2001 et en emploi en 2008, sortis de formation initiale en 1998.

Note de lecture : en $2001,14,8 \%$ des jeunes fonctionnaires de l'échantillon sont déclassés d'un point de vue objectif.

Plus globalement, la différence entre les taux de déclassement des femmes et des hommes en 2001 est significative à $5 \%$; celle des femmes et des hommes en emploi dans la fonction publique en 2008 est significative à $10 \%$; et celle des femmes et des hommes en emploi dans le privé en 2008 est significative à $5 \%$.

(*): Fonction publique.

Tableau 1b. Matrice de passage des hommes - Situation d'embauche pour l'emploi de 2001 situation de 2008 , critère statistique de déclassement (\%)

\begin{tabular}{|l|c|c|c|c|c|c|}
\hline & \multicolumn{7}{|c|}{2008} \\
\hline 2001 & $\begin{array}{c}\text { Fonctionnaires } \\
\text { non déclassés }\end{array}$ & $\begin{array}{c}\text { Fonctionnaires } \\
\text { déclassés }\end{array}$ & $\begin{array}{c}\text { Non fonctionnaires } \\
\text { non déclassés }\end{array}$ & $\begin{array}{c}\text { Non fonctionnaires } \\
\text { déclassés }\end{array}$ & $\begin{array}{c}\text { Privé non } \\
\text { déclassés }\end{array}$ & $\begin{array}{c}\text { Privé } \\
\text { déclassés }\end{array}$ \\
\hline Fonctionnaires non déclassés & 86,6 & 1,7 & 0,4 & 0 & 11,1 & 0,2 \\
\hline Fonctionnaires déclassés & $29,1^{*}$ & 66,7 & 0 & 1,4 & 0 & 2,8 \\
\hline Non fonctionnaires non déclassés & 51,8 & 2,6 & 15,3 & 0 & 27,7 & 2,6 \\
\hline Non fonctionnaires déclassés & 16,9 & 45,3 & 3,8 & 5,7 & 11,3 & 17 \\
\hline
\end{tabular}

Source : Enquête Céreq 2008 auprès de la génération $98,4^{\text {ème }}$ interrogation.

Champ : jeunes hommes en emploi dans la fonction publique en 2001 et en emploi en 2008, sortis de formation initiale en 1998.

Note de lecture : parmi les jeunes hommes fonctionnaires déclassés à l'embauche de leur emploi de 2001, 29,1 \% ne sont plus déclassés en 2008.

Tableau 1c. Matrice de passage des femmes - Situation d'embauche pour l'emploi de 2001 situation de 2008 , critère statistique de déclassement (\%)

\begin{tabular}{|l|c|c|c|c|c|c|}
\hline & \multicolumn{7}{|c|}{2008} \\
\hline 2001 & $\begin{array}{c}\text { Fonctionnaires } \\
\text { non déclassés }\end{array}$ & $\begin{array}{c}\text { Fonctionnaires } \\
\text { déclassés }\end{array}$ & $\begin{array}{c}\text { Non fonctionnaires } \\
\text { non déclassés }\end{array}$ & $\begin{array}{c}\text { Non fonctionnaires } \\
\text { déclassés }\end{array}$ & $\begin{array}{c}\text { Privé non } \\
\text { déclassés }\end{array}$ & $\begin{array}{c}\text { Privé } \\
\text { déclassés }\end{array}$ \\
\hline Fonctionnaires non déclassés & 88,7 & 2,5 & 1,3 & 0,1 & 7,3 & 0,1 \\
\hline Fonctionnaires déclassés & $22,6^{*}$ & 69,2 & 0 & 0,7 & 1,5 & 6 \\
\hline Non fonctionnaires non déclassés & 61,6 & 2,4 & 9,7 & 0,9 & 22,5 & 2,9 \\
\hline Non fonctionnaires déclassés & 22 & 48 & 3,2 & 5,6 & 4,7 & 16,5 \\
\hline
\end{tabular}

Source : Enquête Céreq 2008 auprès de la génération 98,4 ème interrogation.

Champ : jeunes femmes en emploi dans la fonction publique en 2001 et en emploi en 2008, sorties de formation initiale en 1998.

Note de lecture : parmi les jeunes femmes fonctionnaires déclassées à l'embauche de leur emploi de 2001, 22,6\% ne sont plus déclassées en 2008. 
chômage sur les offres d'emploi de niveau inférieur, qui se déclassent ainsi volontairement pour échapper au chômage, les candidats à ces emplois non diplômés se retrouvant eux au chômage. Ainsi cette dégradation des conditions d'emploi des jeunes n'est pas sans effet sur le maintien à un même niveau du taux de déclassement (Nauze-Fichet et Tomasini, 2005).

Le taux de déclassement statistique des hommes, en 2001, atteint près de $14 \%$, inférieur à celui des femmes de plus de trois points. Sept ans plus tard, ce taux ne se réduit que d'un à deux points pour les hommes et les femmes restées en emploi dans la fonction publique. Cette légère diminution du déclassement va de pair avec une importante titularisation des jeunes entre 2001 et 2008 ( $c f$. tableau 1). Sortir de la fonction publique n'assure qu'un déclassement légèrement moindre pour les hommes, les femmes voyant leur situation de déclassement devenir plus fréquentes.

Que cache ce faible reclassement en termes d'évolution de la correspondance formationemploi ? La faible diminution du déclassement ne tient pas au fait qu'entre 2001 et 2008, des associations diplôme-PCS seraient devenues «normales » alors qu'elles ne l'étaient pas. Apparaissent même des évolutions contraire, à savoir des associations diplôme-PCS qualifiées de "normales» en 2001 et de déclassées en 2008 : il s'agit des situations bac + 5 techniciens et bac +5 - professions intermédiaires. Ce sont donc les effectifs de chaque association diplôme-PCS qui évoluent et c'est par le jeu des mobilités individuelles que s'opère le reclassement lorsqu'il a lieu.

Que cache alors ce faible reclassement en termes de mobilité professionnelle?

En premier lieu, en sept années de vie active, le statu quo domine pour les hommes comme pour les femmes : respectivement $71 \%$ et $78 \%$ n'ont pas changé de catégorie socioprofessionnelle entre 2001 et 2008 . Ce résultat tient en partie au niveau d'agrégation important de la nomenclature des PCS retenue. Pour autant, la mobilité ascendante existe et concerne plus fréquemment les hommes que leurs consœurs (17\% contre $12 \%$ respectivement).

En second lieu, les mobilités ascendantes ne vont pas systématiquement de pair avec un reclassement : $30 \%$ de ces mobilités ne s'accompagnent pas d'un reclassement pour les hommes et $19 \%$ pour les femmes. À l'inverse, Statu quo et mobilité descendante ne sont jamais associés à une sortie du déclassement.

La situation initiale des jeunes la plus fréquente en 2001 est celle des fonctionnaires non déclassés pour les hommes comme pour les femmes : autour de $48 \%$ de jeunes sont dans ce cas. Sept ans après, ils représentent deux tiers de l'échantillon pour les deux populations (tableau 2).

Le taux de déclassement observé des fonctionnaires est plus faible que celui des non-titulaires en 2001, et ce de manière un peu plus marquée pour les femmes ( $c f$. tableau 1). En 2008, les non-titulaires n'apparaissent plus davantage déclassés objectivement que 
Tableau 2

Répartition de la population selon le statut, le déclassement statistique en 2001 et en 2008 ( $\%$ en colonne)

\begin{tabular}{|l|c|c|c|c|c|c|}
\hline & \multicolumn{2}{|c|}{ Total } & \multicolumn{3}{c|}{ Femmes } & \multicolumn{2}{c|}{ Hommes } \\
\hline & 2001 & 2008 & 2001 & 2008 & 2001 & 2008 \\
\hline Fonctionnaires non déclassés & 48,8 & 67 & 47 & 67,6 & $52^{*}$ & 66 \\
\hline Fonctionnaires déclassés & 8,5 & 11,3 & 8,7 & 12,1 & 8 & 9,8 \\
\hline Non fonctionnaires non déclassés & 35,3 & 4,8 & 36 & 4,3 & 34,1 & 5,6 \\
\hline Non fonctionnaires déclassés & 7,4 & 0,7 & 8,3 & 1 & 5,9 & 0,5 \\
\hline Privé non déclassés & - & 13,5 & - & 12 & - & 15,9 \\
\hline Privé déclassés & - & 2,7 & - & 3 & - & 2,2 \\
\hline
\end{tabular}

Source : Enquête Céreq 2008 auprès de la génération 98, $4^{\text {ème }}$ interrogation.

Note de lecture : parmi les jeunes hommes en emploi dans la fonction publique en 2001, 52 \% sont fonctionnaires et non déclassés en 2001.

Test de significativité des écarts entre hommes et femmes en 2001 : écart significatif à $1 \%$ pour les fonctionnaires non déclassés, non significatif pour les fonctionnaires déclassés, non significatif pour les non-fonctionnaires non déclassés et significatif à $1 \%$ pour les non-fonctionnaires déclassés.

Test de significativité des écarts entre hommes et femmes en 2008 : écart non significatif pour les fonctionnaires non déclassés, significatif à $1 \%$ pour les fonctionnaires déclassés, significatif à $10 \%$ pour les non-fonctionnaires non déclassés et significatif à $10 \%$ pour les non-fonctionnaires déclassés.

Les chiffres grisés correspondent à des catégories et à des dates pour lesquelles les écarts entre hommes et femmes ne sont pas significatifs.

les fonctionnaires. La titularisation contribuerait-elle à maintenir, voire à conduire à une situation de déclassement?

Lévolution des situations de déclassement et de statut entre 2001 et 2008 montre que les non-titulaires déclassés en 2001 ont plus de chances que les fonctionnaires dans cette situation d'être reclassés sept ans plus tard ; ce reclassement pouvant se réaliser via la sortie de la fonction publique pour les hommes en particulier. Les jeunes non titulaires et déclassés en 2001 deviennent fonctionnaires en restant déclassés pour la majorité (48\% chez les femmes, $45 \%$ chez les hommes - tableaux 1c et $\mathbf{1 b}$ ). L'acquisition du statut associé à un reclassement est plus fréquente chez les femmes (22\%) que chez les hommes (17\%).

Les hommes fonctionnaires déclassés initialement "se reclassent " plus fréquemment que les jeunes femmes dans la même situation de départ (30\% contre $23 \%$ - tableaux $\mathbf{1 b}$, 1c). Enfin, eu sein de la population non titulaire et non déclassée en 2001, les jeunes femmes acquièrent, plus que les jeunes hommes, le statut de fonctionnaire, sans se déclasser pour autant $(62 \%$ contre $52 \%)$.

\section{2. Être et se sentir déclassé : des états qui ne vont pas de pair}

Lorsqu'on choisit de se référer aux ressentis des personnes, on constate une persistance du sentiment de déclassement entre 2001 et 2008. Après plusieurs années passées dans la fonction publique, le sentiment de déclassement devient plus fréquent chez les jeunes 


\section{Graphique 1}

Situation de déclassement statistique et ressenti - Evolution entre 2001 et 2008

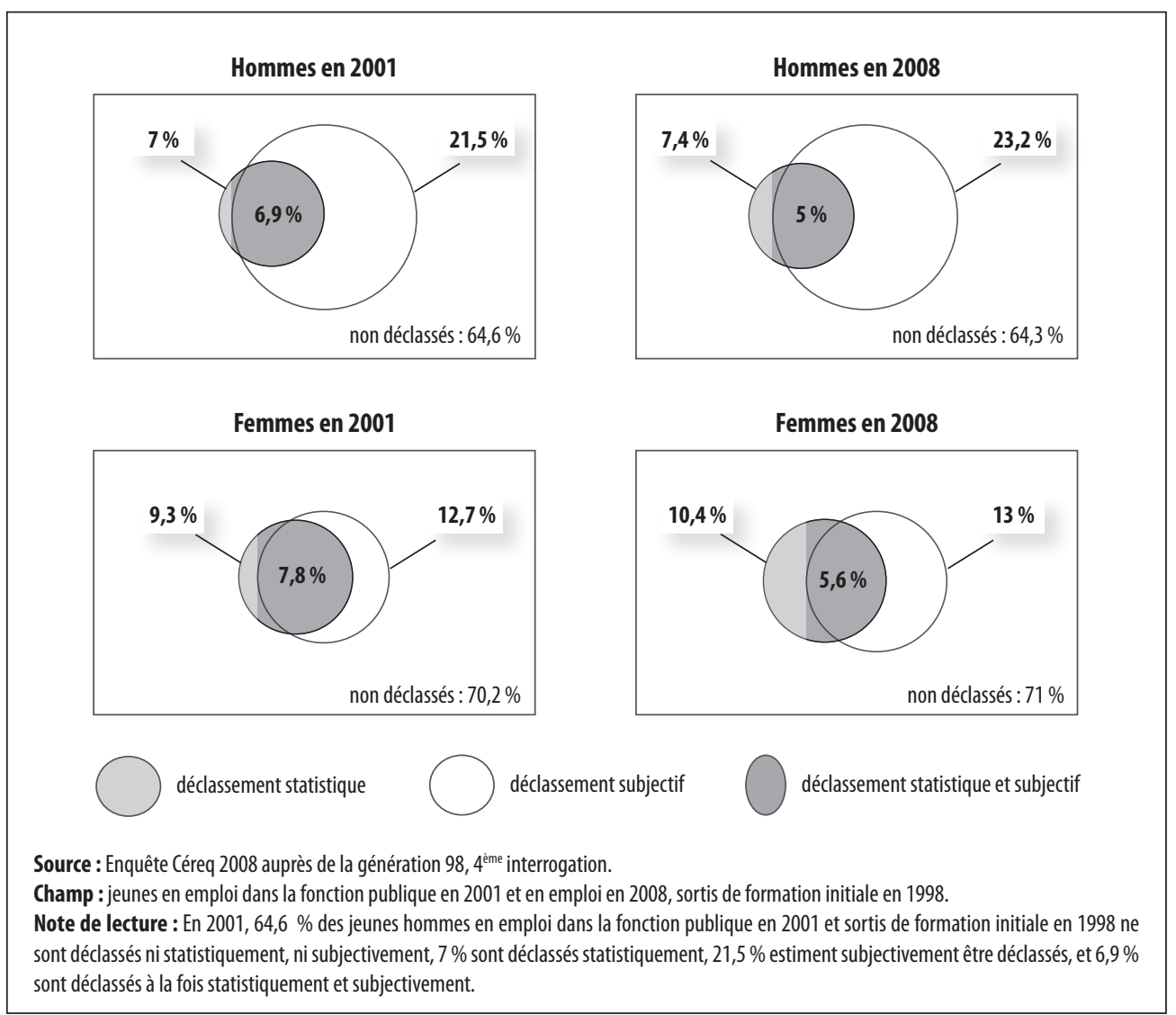

fonctionnaires que chez ceux n'ayant pas acquis ce statut (près de $16,3 \%$ se sentent déclassés en 2008 contre $21,9 \%$ en 2001 chez les titulaires). Un élément d'explication est que, relativement aux jeunes fonctionnaires, les non-titulaires connaissent davantage de promotions sur la période (sur la population considérée, les non-titulaires en 2001 ont trois fois plus de chances de connaitre une promotion entre 2001 et 2008 que les jeunes fonctionnaires en début de période). De plus, quel que soit leur statut, les hommes s'estiment plus souvent déclassés que les femmes et cette différence perdure (près de 10 points d'écart en $2008: 18,3 \%$ des femmes, contre $27,2 \%$ des hommes dans la fonction publique).

Avec l'expérience professionnelle dans la fonction publique, les situations objectives de déclassement et le ressenti varient-ils conjointement?

Les femmes sont plus nombreuses que les hommes, en 2001 comme en 2008, à ne connaitre aucun déclassement (ni statistique, ni subjectif) ( $c f$. graphique 1). Comme vu 


\section{Tableau 3}

\section{Coexistence des déclassements objectif et subjectif, selon le statut d'emploi et le sexe, en 2001 et 2008}

\begin{tabular}{|c|c|c|c|c|c|c|c|c|}
\hline & & & \multicolumn{6}{|c|}{ Déclassement subjectif } \\
\hline & \multirow{2}{*}{\multicolumn{2}{|c|}{$\begin{array}{l}\text { Déclassement } \\
\text { statistique }\end{array}$}} & \multicolumn{2}{|c|}{ Fonctionnaires } & \multicolumn{2}{|c|}{ Non Fonctionnaires FP } & \multicolumn{2}{|c|}{ Privé } \\
\hline & & & Oui & Non & Oui & Non & Oui & Non \\
\hline \multirow{4}{*}{2001} & \multirow{2}{*}{ Oui } & H & $7 \% *$ & $7 \%$ & $7 \%$ & $7 \%$ & & \\
\hline & & $\mathrm{F}$ & $6 \%$ & $9 \%$ & $10 \%$ & $9 \%$ & & \\
\hline & \multirow{2}{*}{ Non } & $\mathrm{H}$ & $19 \%$ & $68 \%$ & $26 \%$ & $60 \%$ & & \\
\hline & & $\mathrm{F}$ & $11 \%$ & $73 \%$ & $14 \%$ & $67 \%$ & & \\
\hline \multirow{4}{*}{2008} & \multirow{2}{*}{ Oui } & $\mathrm{H}$ & $5 \%$ & $8 \%$ & $4 \%$ & $4 \%$ & $4 \%$ & $8 \%$ \\
\hline & & $\mathrm{F}$ & $5 \%$ & $10 \%$ & $4 \%$ & $14 \%$ & $8 \%$ & $12 \%$ \\
\hline & \multirow{2}{*}{ Non } & H & $23 \%$ & $64 \%$ & $13 \%$ & $80 \%$ & $29 \%$ & $59 \%$ \\
\hline & & $\mathrm{F}$ & $13 \%$ & $72 \%$ & $12 \%$ & $70 \%$ & $13 \%$ & $67 \%$ \\
\hline
\end{tabular}

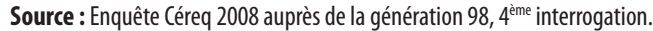

Champ : jeunes hommes en emploi dans la fonction publique en 2001 et en emploi en 2008, sortis de formation initiale en 1998.

(*) Note de lecture : parmi les jeunes hommes fonctionnaires en 2001, 7 \% sont statistiquement et se sentent déclassés.

précédemment, elles sont un peu plus nombreuses que les hommes à être statistiquement déclassées, moins à avoir le sentiment de l'être, et ce, aux deux dates.

La situation de double déclassement (être statistiquement et se sentir déclassé) révèle un état de conscience de la situation statistique plus grand pour les hommes que pour les femmes : en 2008, $40 \%{ }^{9}$ des hommes déclassés statistiquement ont en même temps le sentiment de l'être ; seulement $35 \%$ des femmes ont cet état de conscience. Ils sont également plus nombreux que les femmes à se sentir déclassés sans l'être statistiquement. En effet, parmi les jeunes hommes se déclarant déclassés en 2008, 82 \% ne le sont pas statistiquement ; cette proportion n'est plus que de $70 \% \mathrm{chez}$ les femmes. Le temps ne permet pas de prendre davantage conscience de la situation statistique pour les deux sexes. En effet, en 2001, la "proportion des conscients " n'est pas moindre qu'en 2008 ; ils ne sont pas moins nombreux en 2008 à se sentir déclassés alors qu'ils ne le sont pas statistiquement. L'expression du sentiment de déclassement n'est pas en concordance systématique avec la situation effective d'inadéquation entre le niveau de l'emploi et le niveau de formation ; cet indicateur subjectif peut donc aussi traduire d'autres mécontentements, dont l'absence de réalisation professionnelle ${ }^{10}$.

L'absence de titularisation ne participerait-elle pas à cette non-concordance des déclassements statistiques et subjectifs ? Autrement dit, ne pas être fonctionnaire, en particulier

$9.5 \% /(5 \%+7,4 \%)=40,3 \%$ - cf. graphique 1, Hommes 2008 .

10. Réalisation professionnelle et déclassement subjectif renvoyant au plus ou moins bon usage fait des compétences sont deux dimensions distinctes de la satisfaction professionnelle et s'expriment de manière similaire pour les hommes et les femmes. 


\section{Tableau 4}

Probabilité de se sentir déclassé en 2001 et 2008 selon la situation statistique de déclassement Effet du sexe (Odds ratio-Modèle Logit)

\begin{tabular}{|c|c|c|c|c|}
\hline \multirow[b]{2}{*}{ Se sentir déclassé en 2001} & \multicolumn{2}{|c|}{2001} & \multicolumn{2}{|c|}{2008} \\
\hline & - & & 3.605 & $(0.405)$ \\
\hline $\begin{array}{l}\text { Femme statistiquement déclassée } \\
\text { Femme statistiquement non déclassée } \\
\text { Homme statistiquement déclassé } \\
\text { Homme statistiquement non déclassé }\end{array}$ & $\begin{array}{l}2.7(£) \\
0.7 \\
3.1 \\
\text { Ref. }\end{array}$ & $\begin{array}{l}(0.492) \\
(0.091) \\
(0.695)\end{array}$ & $\begin{array}{c}1.7 \\
0.7 \\
2 \\
\text { Ref. }\end{array}$ & $\begin{array}{l}(0.323) \\
(0.092) \\
(0.469)\end{array}$ \\
\hline $\begin{array}{l}\text { Fonctionnaire } \\
\text { Non fonctionnaire } \\
\text { Secteur privé }\end{array}$ & $\begin{array}{l}0.8^{*} \\
\text { Ref. }\end{array}$ & $(0.085)$ & $\begin{array}{c}1.8^{*} \\
\text { Ref. } \\
1.817^{*}\end{array}$ & $\begin{array}{l}(0.467) \\
(0.503)\end{array}$ \\
\hline $\begin{array}{l}\text { Avoir un enfant ou plus } \\
\text { Vivre en couple }\end{array}$ & $\begin{array}{l}1.5 \\
0.8^{*}\end{array}$ & $\begin{array}{l}(0.235) \\
(0.093)\end{array}$ & $\begin{array}{l}\text { ns } \\
\text { ns }\end{array}$ & \\
\hline Ancienneté dans l'emploi (mois) & ns & & $1^{*}$ & $(0.008)$ \\
\hline Expérience professionnelle antérieure (mois & 1 & $(0.008)$ & $1^{*}$ & $(0.005)$ \\
\hline \multicolumn{5}{|l|}{ Type de fonction publique: } \\
\hline $\begin{array}{l}\text { Education nationale } \\
\text { Fonction publique territoriale } \\
\text { Fonction publique hospitalière } \\
\text { Autre fonction publique d'État }\end{array}$ & $\begin{array}{c}\text { Ref. } \\
\text { ns } \\
0.4 \\
\text { ns }\end{array}$ & $(0.084)$ & $\begin{array}{l}- \\
- \\
-\end{array}$ & \\
\hline \multicolumn{5}{|l|}{ Niveau de formation initiale (\$): } \\
\hline $\begin{array}{l}\text { Niveau I } \\
\text { Niveau II } \\
\text { Niveau III } \\
\text { Niveau IV sup } \\
\text { Niveau IV } \\
\text { Niveau V } \\
\text { Niveaux Vbis et VI }\end{array}$ & $\begin{array}{c}\text { ns } \\
\text { ns } \\
\text { ns } \\
\text { ns } \\
2.6^{*} \\
\text { ns } \\
\text { Ref. }\end{array}$ & $(1.241)$ & $\begin{array}{c}0.4^{*} \\
0.4^{*} \\
0.4^{*} \\
\text { ns } \\
\text { ns } \\
\text { ns } \\
\text { Ref. }\end{array}$ & $\begin{array}{l}(0.154) \\
(0.180) \\
(0.159)\end{array}$ \\
\hline \multicolumn{5}{|l|}{ Spécialités de formation: } \\
\hline $\begin{array}{l}\text { Tertiaires } \\
\text { Industrielles } \\
\text { Générales }\end{array}$ & $\begin{array}{l}\text { ns } \\
\text { ns } \\
\text { Ref. }\end{array}$ & & $\begin{array}{l}\mathrm{ns} \\
1.6 \\
\text { Ref. }\end{array}$ & $(0.255)$ \\
\hline LogVraisemblance & \multicolumn{2}{|c|}{$-1168,07$} & \multicolumn{2}{|c|}{$-1146,83$} \\
\hline$N$ & \multicolumn{2}{|c|}{2421} & \multicolumn{2}{|c|}{2421} \\
\hline
\end{tabular}

Source : Enquête Céreq 2008 auprès de la génération $98,4^{\text {ème }}$ interrogation.

(£) Note de lecture : Une femme déclassée d'un point de vue statistique en 2001 a 2,7 fois plus de chances de se sentir déclassée à cette date qu'un homme non statistiquement déclassé mais qui a, par ailleurs, les mêmes caractéristiques (même employeur et statut, même ancienneté et expérience professionnelle, même niveau et spécialité de formation). Ecart type entre parenthèses.

$\left.{ }^{*}\right)$ Note : les paramètres présentés sont significatifs au seuil de $5 \%$; ceux avec une étoile le sont au seuil de $10 \%$; sinon, les paramètres non significatifs au seuil de $10 \%$ sont notés «ns».

(\$) : Niveau I : sorties avec un diplôme de troisième (DEA ou DESS : respectivement diplôme d'études approfondies et diplôme d'études supérieures spécialisées), cycle universitaire ou un diplôme de Grande École) ; Niveau II : sorties avec un diplôme de second (licence ou maîtrise) cycle universitaire ; Niveau III : sorties avec un diplôme de niveau Bac + 2 ans (DUT, BTS, DEUG - respectivement diplôme universitaire de technologie, brevet de technicien supérieur et diplôme d'études universitaires générales -, écoles des formations sanitaires ou sociales, etc.) ; Niveau IV secondaire : sorties des classes terminales du second cycle long ; Niveau IV Sup : abandons des scolarisations post-baccalauréat avant d'atteindre le niveau III ; Niveau V : sorties de I'année terminale des cycles courts professionnels et abandons de la scolarité du second cycle long avant la classe terminale ; Niveau V Bis : sorties de 3ème générale, de 4ème et 3ème technologiques et des classes du second cycle court avant l'année terminale ; Niveau VI : sorties du 1er cycle du second degré (6ème, 5ème, 4ème) et des formations préprofessionnelles en un an. 
après plusieurs années passées en emploi dans la fonction publique, pourrait conduire à l'expression d'une insatisfaction traduite au travers du sentiment de déclassement. En 2001, la part des jeunes se sentant déclassés sans l'être statistiquement est plus importante chez les non-titulaires, comparés aux fonctionnaires ( $c f$. tableau 3) : $14 \%$ des femmes non fonctionnaires, en 2001, se sentent et ne sont pas déclassées, contre $11 \%$ parmi les fonctionnaires (ces parts sont respectivement de $26 \%$ et $19 \%$ pour les hommes). En 2008, les femmes non déclassées statistiquement expriment aussi fréquemment un sentiment de déclassement, qu'elles soient titulaires ou non ( $13 \%$ pour les fonctionnaires et $12 \%$ pour les non-titulaires). A contrario, les hommes fonctionnaires expriment, plus souvent que les non-titulaires, un sentiment de déclassement alors qu'ils ne sont pas déclassés ( $23 \%$ contre $13 \%)$. Ce résultat confirme que le statut de fonctionnaire ne préserve pas de l'insatisfaction professionnelle, notamment pour les hommes ${ }^{11}$.

Lévolution du sentiment de déclassement ne suit pas nécessairement l'évolution de la situation statistique. Il convient de cerner le rôle propre du sexe selon la situation statistique de déclassement sur l'expression du sentiment ; à cet effet, pour considérer son évolution ${ }^{12}$, nous menons une analyse toutes choses égales par ailleurs, en 2001 comme en 2008.

En 2001, comme sept ans plus tard, la situation statistique de déclassement contribue logiquement à augmenter le ressenti pour les deux sexes. À ces deux dates, les hommes expriment un sentiment de déclassement plus souvent que leurs consœurs, toutes choses égales par ailleurs, y compris le fait d'être fonctionnaire. L'effet de ce dernier facteur change entre 2001 et 2008 : si être fonctionnaire réduit le sentiment de déclassement en début de vie active, il contribue à son accroissement après dix années sur le marché du travail. Enfin, le sentiment de déclassement est persistant ; en témoigne l'effet important, toutes choses égales par ailleurs, du sentiment initial. De même, le temps passé en emploi contribue légèrement à accentuer le sentiment.

S'agissant des variables relatives à la situation familiale, elles n'influent le ressenti de déclassement qu'en début de parcours professionnel. En effet, avoir un enfant en 2001 aggrave ce sentiment, alors que vivre en couple conduit à le modérer. Le type de fonction publique qui emploie les jeunes en 2001 ne joue aucun rôle dans l'expression de leur ressenti, à l'exception de la fonction publique hospitalière qui contribue à restreindre fortement le ressenti. L'accès réglementé aux professions dans ce secteur peut expliquer ce résultat. En 2008, parmi les jeunes passés dans le secteur privé, le ressenti ne s'en trouve pas pour autant diminué. À déclassement statistique donné, il ne semble pas exister globalement d'effet propre aux niveaux et aux spécialités de formation sur le sentiment de déclassement des jeunes.

11. Ces constats sont obtenus en moyenne et ne résultent pas d'analyse de dynamiques individuelles.

12. Compte tenu de la faible taille de l'échantillon des jeunes déclassés initialement - en 2001 - nous ne pouvons estimer les paramètres des facteurs explicatifs du reclassement. Nous proposons dès lors de confronter des estimations portant sur les situations de 2001 et 2008 conduites séparément. 


\section{Conclusion}

La question centrale de cet article est de savoir dans quelle mesure les jeunes qui entament une carrière dans la fonction publique "rattrapent ", avec l'ancienneté accumulée dans ce secteur d'emploi, leur éventuel déclassement à l'embauche. En particulier, sur ce marché du travail spécifique, les jeunes femmes sont-elles pénalisées ou non, du seul fait de leur sexe, en matière de reclassement?

L'ensemble des résultats permet de dresser un bilan sur le rattrapage des situations de déclassement. Ils soulignent globalement à la fois l'existence d'une inertie dans les situations de déclassement statistique, et ce plus particulièrement pour les femmes, et un reclassement légèrement moins fréquent pour les jeunes femmes, laissant penser que le déclassement constitue pour elles un plancher collant.

La sortie du déclassement est davantage possible pour les non-titulaires que pour les fonctionnaires. Il existe un double mouvement de reclassement et acquisition du statut de fonctionnaire qui est davantage l'apanage des femmes; les hommes non fonctionnaires en début de vie active se reclassent quant à eux à la fois par le biais de la titularisation mais aussi en rejoignant le secteur privé.

Enfin, l'accès au statut de fonctionnaire ne s'opère pas au prix d'un déclassement (statistique) : pour les deux sexes, les jeunes qui, en devenant fonctionnaires entre l'emploi occupé en 2001 et 2008, acceptent de se déclasser, constituent des cas marginaux.

De plus, on met en évidence que les femmes s'estiment toujours moins déclassées que leurs confrères. Si en 2001, les fonctionnaires expriment moins souvent le sentiment de déclassement que les autres, en 2008, ils deviennent les plus nombreux à se déclarer insatisfaits.

Pour traiter du " rattrapage ", il convient de se baser sur l'évolution de la situation statistique de déclassement et non sur sa mesure subjective ; en effet, le ressenti d'un désajustement entre niveau de formation et niveau d'emploi peut disparaître dans le temps du seul fait de "l'accoutumance ", ou apparaître car il peut traduire une insatisfaction professionnelle bien plus large, sans qu'aucune modification de la situation statistique ne soit observée.

Pour autant, la confrontation des indicateurs statistique et subjectif révèle la non-correspondance des deux dimensions qu'ils recouvrent. Plus des deux tiers des individus déclassés statistiquement en 2008 n’ont pas conscience de l'être. De manière symétrique, à cette date, plus des trois quarts de la population qui se déclare déclassée ne l'est pas au regard de l'indicateur statistique retenu.

Selon Sicherman (1991), les individus en situation de déclassement statistique devraient connaitre, plus probablement que les autres, une mobilité ascendante. Cette hypothèse est ici mise à mal ; en effet, malgré l'acquisition d'une expérience professionnelle spécifique, une grande majorité des jeunes initialement déclassés ne connait pas de mobilité 
ascendante permettant le rattrapage du décalage initial. C'est même plutôt l'hypothèse d'une faible évolution de la structure d'emploi ne compensant jamais la structure des diplômes des salariés (Tsang et Levin, 1985) qui semble prévaloir, faisant du déclassement un phénomène durable plutôt que transitoire. Cette interprétation reste à relativiser car sur cette période la conjoncture économique s’est fortement dégradée. Autrement dit, les mobilités promotionnelles auraient été certainement plus nombreuses dans un contexte économique meilleur. Reste que, dans l'inertie des situations de déclassement, on ne peut évaluer ce qui relève des freins à la mobilité liés à la conjoncture.

Dès lors, les femmes qui ont choisi d'entrer dans la fonction publique, au prix d'un déclassement éventuel qu'elles ne rattraperont pas, ou moins que les hommes, auraient-elles mieux fait de se tourner vers le secteur privé ? Répondre à cette question suppose de comparer les situations des femmes en emploi dans le secteur privé avec celles de la fonction publique (et non pas homme/femme). Cette comparaison ne sera pertinente que si l'on tient compte des mécanismes d'auto sélection ${ }^{13}$ des individus pour l'un ou l'autre des secteurs, le choix des femmes pour la fonction publique les préservant relativement de conditions d'emploi globalement moins favorables que celles des hommes. Parallèlement, étant donné les mécanismes de sélection à l'œuvre ${ }^{14}$, la situation des hommes dans la fonction publique est peut être moins favorable que celle des hommes dans le secteur privé (comparaison hommes du public/hommes du privé).

\section{Bibliographie}

Affichard J. (1981), "Quels emplois après l'école : la valeur des titres scolaires depuis 1973 ? ", Economie et Statistique, n 134, pp. 7-27.

Audier F., di Paola V., Lambert M., Meurs D., Moullet S., Perez C. (2004), Les déterminants des entrées dans les fonctions publiques : parcours du combattant ou pis-aller?, Rapport pour le ministère de la Recherche, ACI Travail dans la Fonction publique.

Battu H., Belfield C.R. and Sloane P.J. (2000), "How well can we measure graduate over-education and its effets?", National Institute Economic Review.

13. Le choix de la fonction publique est loin d'être aléatoire : certains attributs individuels, dont l'aversion pour les incertitudes du marché du travail privé, peuvent expliquer, notamment pour les femmes, les décisions de se porter sur le marché du travail de la fonction publique ( $c f$. de Singly et Thélot, 1988).

14. On a notamment montré que les jeunes hommes qui entrent dans la fonction publique connaissent des trajectoires plus précaires que lorsqu'ils s'insèrent dans le secteur privé, le choix de la fonction publique s'apparentant pour eux, plus que pour leurs consœurs, à un pis-aller (di Paola et Moullet, 2004). Les hommes en emploi dans la fonction publique ont en conséquence des caractéristiques - une aversion relative pour le risque, une motivation pour le service public par exemple - qui, justifiant ce choix, les distinguent des salariés du secteur privé. 
Baudelot C. et Glaude D. (1989), "Les diplômes se dévaluent-ils en se multipliant ? ", Economie et Statistique, n ${ }^{\circ} 225$, pp. 3-15.

Baudelot C., Gollac M., Bessière C., Coutant I., Godechot O., Serre D. et Viguier F. (2003), Travailler pour être heureux? Le bonheur et le travail en France, Fayard Ed. Paris.

di Paola V. et Moullet S. (2009), «Femmes et fonction publique : un risque calculé de déclassement?", Travail et Emploi, n 120, novembre-décembre.

di Paola V. et Moullet S. (2004), «L'emploi public et les trajectoires d'insertion des jeunes ", Economie et Statistique, n³69-370.

di Paola V., Moullet S. et Vero J. (2005), " Le déclassement dans les Fonctions publiques d'Etat et territoriales ", dans Des Formations pour quels emplois ?, GIRET J.-F., Lopez A., Rose J. (eds.), La Découverte-Céreq, "Recherche ».

Elster J. (1983), Sour Grapes, Cambridge University Press.

Forgeot G. et Gautié J. (1997), «Insertion professionnelle des jeunes et processus de déclassement ", Document de travail de l'Insee-Série rouge, avril, n 9711, 40 p.

Fougère D. et Pouget J., (2004), «Les déterminants économiques des entrées dans la fonction publique ", Economie et Statistique, n 369-370.

Giret J.-F. (2005), «Quand les jeunes s'estiment déclassés », dans Des Formations pour quels emplois ?, Giret J.-F., Lopez A., Rose J. (eds.), La Découverte-Céreq, " Recherche », pp. 279-288.

Giret J.-F. et Lemistre P. (2004), « Le déclassement à l'embauche des jeunes : vers un changement de la valeur des diplômes ?", Cahiers économiques de Bruxelles - Brussels economic review, special issue in Economics of Education and Human Ressources, vol. 47, $\mathrm{n}^{\circ} 3$, pp. 483-503.

Giret J.-F., Nauze-Fichet E. et Tomasini M. (2006), «Le déclassement des jeunes sur le marché du travail ", Données sociales - La société française, pp. 307-314.

Hartog J. (1999), "Suréducation et sous-éducation dans une perspective de formation professionnelle ", Revue européenne de formation professionnelle, $\mathrm{n}^{\circ}$ 16, pp. 53-58, Mesure subjective du déclassement.

Mc Cormick B. (1990), "A theory of signalling during job search, employment efficiency, and stigmatised job”, Review of Economics Studies, 57, pp. 299-313.

Marry C. (2005), Enquête sur les promotions CR-DR dans une section des sciences de la vie du CNRS, Rapport de recherche pour la Direction générale du CNRS et la Mission pour la place des femmes au CNRS (dir), juin, $102 \mathrm{p}$. 
McDowell J.M., Singell L.D. and Ziliak J.P. (1999), "Cracks in the glass ceiling: Gender and promotion in the economics profession", American Economic Review, 89(2).

Nauze-Fichet E. et Tomasini M. (2005), " Parcours des jeunes à la sortie du système éducatif et déclassement salarial ", Economie et Statistique, n 388-389, pp. 57-83.

Nauze-Fichet E. et Tomasini M. (2001), « Diplôme et insertion sur le marché du travail : approches socioprofessionnelle et salariale du déclassement ", Economie et Statistique, $\mathrm{n}^{\circ} 304$, pp. 21-43.

Observatoire de l'emploi public, Rapport annuel 2004-2005 : http://www.fonctionpublique.gouv.fr/article622.html.

RubB S. (2003), “Overeducation: a short or long run phenomenon for individuals?" Economics of Education Review, vol. 22, pp. 389-394.

Sen A.K. (1992), Inequality Reexamined, Oxford University Press, Traduction française : Repenser l'inégalité, Seuil (2000).

Sicherman N. (1991), "Overeducation in the Labor Market", Journal of Labor Economics, $\mathrm{n}^{\circ} 9$, pp. 101-122.

Singly (de) F. et Thélot C. (1988), Gens du privé, gens du public, Dunod.

Tsang M.C. and Levin H.M. (1985), "The Economics of Overeducation”, Economics of Education Review, Vol. 4/2, pp. 93-104.

Tsang M.C., Levin H.M., Rumberger R.W (1991), “The impact of surplus schooling on work productivity", Industrial Relations, n 30, pp. 209-228.

Vinokur A. (1995), "Réflexions sur l'économie du diplôme ", Formation Emploi, n 52, oct-déc., pp. 151-183. 